\title{
A Mathematical model for quantifying workers' learning range on repetitive construction projects
}

\author{
Mohammad Rahal ${ }^{\mathrm{a}}$, Hiam Khoury ${ }^{\mathrm{a}, *}$
}

${ }^{a}$ American University of Beirut, Riad El Solh 1107 2020, Beirut P.O. Box 11-0236, Lebanon

\begin{abstract}
The role of planning and management in the construction industry has always been significant towards improving project schedules. However, the impact of planning strategies on different construction schedules poses a margin for development and enhancement, especially when it comes to managing linear schedules or schedules of projects undergoing repetitive types of work. Previous research efforts developed models to individually analyze the influence of congestion and the learning curve factors on linear schedules, but failed to capture the combined complexities and dynamics when integrating both. Therefore, this paper puts forward the groundwork of a scheduling optimization framework and presents work targeted at quantifying the learning development range of construction workers on repetitive projects. The ultimate goal is to minimize potential congestions by taking into account the inherent uncertainties of linear activities while considering the learning curve effect. More specifically, three dimensions of uncertainty are considered for each activity, namely at the level of the activity itself, at the level of the activity and its predecessors, and at the activity-network level. At the heart of the proposed mathematical model is a fuzzy-based system that generates a minimum percentage reduction in productivity boundaries for each activity with different uncertainty dimensions. The presented fuzzy system will, in future work, become the foundation of a time-cost optimization framework for linear scheduling methods.
\end{abstract}

(C) 2019 The Authors. Published by Budapest University of Technology and Economics \& Diamond Congress Ltd.

Peer-review under responsibility of the scientific committee of the Creative Construction Conference 2019.

Keywords: Constructio;, Linear Scheduling, Productivity ; Learning Curve; Fuzzy Logic

\section{Background}

The learning phenomenon states that the time and effort required to complete any task decreases as the number of repetitions increases and further work experience is gained (Lam, Lee, \& $\mathrm{Hu}, 2001$ ). This concept, known as the learning curve effect, has initially originated in the airplane manufacturing industry (Wright, 1936). Subsequently, applications of this concept has spread through other sectors of manufacturing until reaching different areas in the construction industry (Hamade, Jaber, \& Sikström, 2009; Hinze \& Olbina, 2009; Lam et al., 2001; Wong, On Cheung, \& Hardcastle, 2007; Zhang, Zou, \& Kan, 2014). Other research works also considered the impact of the learning effect on different site activities such as prestressed concrete and piles (Hinze \& Olbina, 2009), rebars Jarkas, 2010), formwork ( Jarkas \& Horner, 2011), and caisson construction (Panas \& Pantouvakis, 2013).

More specifically, some research efforts incorporated the learning curve effect within scheduling techniques applied for repetitive projects, or what is called the line-of-balance (LOB) technique (Suhail \& Neale, 1994). The aim was to enhance the resulting schedules that are based on the false assumption of constant production rates while ignoring the 
Mohammad Rahal, Hiam Khoury/ Proceedings of the Creative Construction Conference (2019) 074 https://doi.org/10.3311/CCC2019-074

effect of learning (Arditi, Tokdemir, \& Suh, 2002). Other works have targeted this improved scheduling approach and included the effect of learning on LOB scheduling (Arditi, Tokdemir, \& Suh, 2001), the obstacles and potentials when considering the learning effect in LOB scheduling (Zahran, Nour, \& Hosny, 2016), and the modeling of the LOB scheduling with a learning development effect (Ammar \& Abdel-Maged, 2018). Besides, many research works mentioned that maintaining work continuity in repetitive projects is recommended to minimize disruption and maximize the benefits of the learning curve (Ammar, 2012; Ammar \& Elbeltagi, 2001; Elbeltagi, Elkassas, Rasheed, \& Al-Tawil, 2007; Moselhi \& El-Rayes, 1993; Thabet \& Beliveau, 1994). Another recent effort worked on enhancing the precedence diagramming method by introducing two new precedence relationships (point-to-point and continuous relations) and while assigning either linear, non-linear, or learning curve production-time functions to activities (Hajdu, 2015; Hajdu, 2016). However, the author assumed a fixed production-time function for each activity without accounting for uncertainties that control their production variation range typically encountered in construction.

As such, despite the importance and the relevance of the learning curve effect in scheduling, in particular linear scheduling or LOB, the aforementioned literature addressing this matter is either ignoring the effect of learning, or assuming fixed or maximum learning in the productivity rates (Tokdemir, Arditi, \& Balcik, 2006). Therefore, in order to enhance linear scheduling methods, there is a need to include a range of learning rates for each activity that best describes the maximum and minimum productivity boundaries. In other words, it is crucial to study the dimensions of uncertainty lying within each activity which, in turn, can directly impact the overall schedule. Therefore, this study defines three dimensions describing: (1) the flexibility of the activity itself, (2) the influence of the activity location on the project network, and (3) the overlapping impact of the activity on its corresponding predecessors due to the learning curve effect. As such, instead of only considering maximum learning rates, a synthesized degree of uncertainty, as explained above, can yield a minimum percentage reduction in the productivity boundaries defined by a decreased range of allowable learning rates. This process is made possible, in this study, through a fuzzy inference system (Garibaldi \& John, 2003; Sivanandam, Sumathi, \& Deepa, 2007) that appears as an attractive alternative for studying the impact of activities' uncertainty on productivity variations, and thus the overall schedule. Fuzzy logic is suitable for such a problem where there are incomplete or imprecise relationships between the variables under study (Chan, Chan, \& Yeung, 2009). In this case, the proposed mathematical fuzzy system takes the activity's uncertainty dimensions as inputs and outputs a minimum percentage reduction in the original range of minimum and maximum productivity rates.

\section{Proposed Model}

\subsection{Describing Activities' Allowable Learning Development Range}

Prior to presenting the proposed mathematical fuzzy system and detailing its various elements, it is worth describing first the allowable learning development range for a certain construction activity. By looking at linear activities separately, each activity has a range of minimum and maximum productivity rates defined by the zero-learning rate (i.e. 100\%) and the maximum learning rate, respectively. Figure 1 illustrates the original productivity boundaries for a certain construction activity, in particular the minimum productivity rate (line $\mathrm{OA}$ ) and the maximum productivity rate (line $\mathrm{OB}$ ). In this case, OA is assumed to have a constant optimized productivity rate with no learning. However, the curve corresponding to the maximum learning rate $(\mathrm{OB})$ is assumed to be an average linear straight line following the Crawford's incremental unit time model (Jaber, 2016). For ease of calculation, the incremental time at the maximum learning rate of the Crawford's algebraic midpoint is considered the maximum productivity rate for all iterations and the cumulative average timeout at point B is computed using the learning curve function adopted from Malyuz and Pem (2013). As such, linear activities are governed by various project-based, activity-based, and interaction-based factors that can affect their productivity ranges. This thereby requires determining a new acceptable range (OA' - OB') within the original range of learning rates to account for all the inherent schedule uncertainties. The shaded area in Figure 1 indicates that the triangle $\mathrm{OAB}$, bound by the original minimum and maximum learning rate curves, is minimized to a new allowable learning development region illustrated by the area OA'B'. 
Mohammad Rahal, Hiam Khoury/ Proceedings of the Creative Construction Conference (2019) 074 https://doi.org/10.3311/CCC2019-074

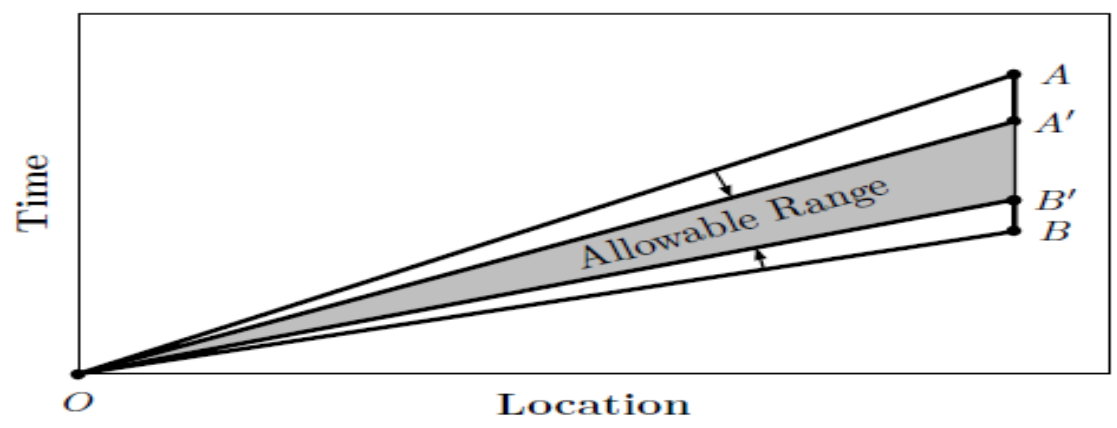

Figure 1: Space-time float of an activity

\subsection{Presenting the Proposed Fuzzy Inference System Mechanism}

Figure 2 illustrates the proposed mathematical fuzzy system.

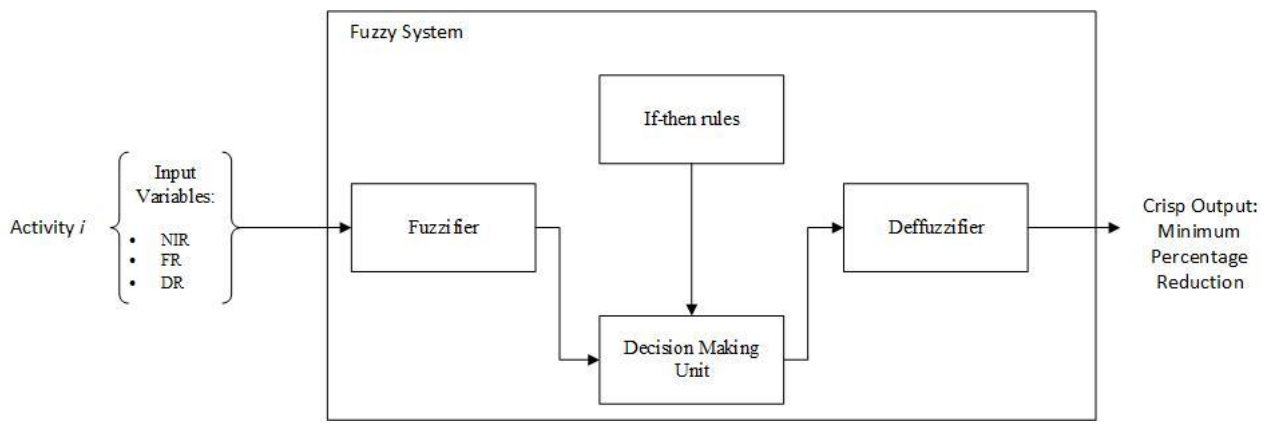

Figure 2: Fuzzy Inference System Mechanism

As shown in Figure 2, the impact level of an activity on the project network (i.e. NIR), the activity flexibility (i.e. FR), and the activity-activity overlap (i.e. DR) form the input variables (or attributes) of the fuzzy inference system, and directly affect the output. The output is actually the minimum percentage reduction in the area residing between the initial productivity boundary lines (Figure 1 ) and is quantified as follows:

$$
\%=\frac{A_{O A B}-A_{O A^{\prime} B^{\prime}}}{A_{O A B}}=1-\frac{A_{O A^{\prime} B^{\prime}}}{A_{O A B}}
$$

Another system component includes the fuzzifier which takes in the crisp inputs and converts them into a fuzzy number $<0,1\rangle$ using its respective membership function. A membership function (MF) is a curve that mathematically defines the degree of an element's membership in a fuzzy set. The input variables are assigned degrees of membership in various classes or linguistic term sets (Wang, 2015). In this study, the suggested three input variables can have values of high, medium and low depending on the ratio. The subjective measures of these linguist terms are defined using either triangular or trapezoidal-shaped membership functions. After defining the fuzzifier component, the next step is to define the logical statements or the if-then rules. Fuzzy inference rules map input to output by feeding the decisionmaking unit of the fuzzy system with the ingredients to formulate decisions (Sivanandam et al., 2007). Typically, this intelligent component unit (Figure 2) assumes one of two formats in any fuzzy system, Takagi-Sugeno or Mamdani. Since the input in our system is intuitive and is expected to be provided by construction engineering experts, the Mamdani paradigm is applied (Sivanandam et al., 2007). Unlike Takagi-Sugeno, the output of a Mamdani fuzzy-based system is also fuzzy and a defuzzifier is thereby needed as depicted in Figure 2. Therefore, the output of the ruling process is defuzzified by converting it into a numerical format to generate numerical probabilities (i.e. percentages). For simplicity purposes, the defuzzification method used within this system is the Centroid of Area method (Wang, 2015). It was selected since it returns estimates right at the center of the membership function curve; not too low or too high. The following subsection describes in detail the three input variables. 
Mohammad Rahal, Hiam Khoury/ Proceedings of the Creative Construction Conference (2019) 074 https://doi.org/10.3311/CCC2019-074

\subsection{Defining the Fuzzy Input Variables}

\subsubsection{Network Influence Ratio (NIR)}

The network influence ratio implies the relevance of a particular activity with other activities in the project network. It gives an indication about the degree of influence of a certain activity on the overall schedule. An activity's uncertainties and resulting delays and distortions are more likely to influence its succeeding activities and cause project delays, especially when the activity has a great number of successors. Thus, a larger percentage reduction in the productivity range is desirable for this activity. This ratio is measured as follows:

$N I R_{i}=\frac{N s_{i}}{N s_{i}+N p_{i}}$

Where, $\mathrm{N}_{\mathrm{S}}$ is the total number of successors (direct and indirect) of Activity $\mathrm{i}$, and $\mathrm{Np}$ is the total number of predecessors (direct and indirect) of activity i. NIR returns a value of 1 for the first activity as it has the highest number of successors and thereby impacts the schedule the most, and 0 for the last activity which has no successors.

\subsubsection{Flexibility Ratio $(F R)$}

This ratio is an input variable that is directly related to the space-time floats of linear activities and represents the flexibility of a particular activity. As previously mentioned, productivity boundaries are defined by the minimum and maximum learning rates and are used to generate a space-time float triangle for that activity (Figure 1). Furthermore, the most likely learning rate is crew-dependent, results in a productivity rate that lies somewhere between the activity boundaries, and its location implies how flexible an activity is. In other words, the closer the most likely productivity rate to the maximum productivity rate, the more flexible the activity. Considering that the project is scheduled with no learning for all linear activities, the most likely learning rates being far-off from the zero-learning rate can offer a greater amount of leeway for that activity. It has been found through previous studies that activities with less flexibility are less likely to be completed on time (Yang, Fu, Li, Huang, \& Peng, 2008), and therefore less percentage reduction is required for these activities. Consequently, when the activity $i$ has a low flexibility, it imposes a higher risk on the time completion of succeeding activities and accordingly requests a smaller percentage reduction of the space-time float. In contrast, a greater percentage reduction is witnessed for more flexible activities as they are less likely to delay succeeding activities. The value of the FR for Activity i can be determined as shown below:

$F R_{i}=\frac{P_{\operatorname{most}, i-} P_{\min , i}}{P_{\max , i-} P_{\min , i}}$

Where, $\mathrm{P}_{\min }$ is the optimum productivity at zero-learning, $\mathrm{P}_{\max }$ is the optimum productivity at maximum learning rate, and $\mathrm{P}_{\text {most }}$ is the optimum productivity at the most likely learning rate

\subsubsection{Dependency Ratio (DR)}

The importance of this ratio is manifested when the learning curve effect comes into play. It captures the degree of interference of the current activity with its corresponding predecessors, if it is performing work at a maximum productivity. In other words, it is important to identify the impact that an activity operating at a maximum learning rate has on its predecessor's learning development range. Due to repetition in linear projects, different activities operate at different locations simultaneously, and potential congestion may be created as a result of any deviation from planned productivity rates. Besides, the learning curve effect is a major factor causing productivity deviations in repetitive projects. Figure 3 presents the space-time floats for activities 1 and 2 each bound by their minimum and maximum productivity rate lines. More specifically, Activity 1 is bound by lines OA and OB while the productivity range of activity 2 lies within EC and ED. At zero learning, activity 1 (OA) and activity two 2 (EC) have a finish-to-finish relationship and an associated lag. At the maximum learning rate (MaxLR), the dotted productivity line (ED) of activity 
Mohammad Rahal, Hiam Khoury/ Proceedings of the Creative Construction Conference (2019) 074 https://doi.org/10.3311/CCC2019-074

2 intersects with the minimum productivity line (zero-learning) of its predecessor (i.e. OA). As shown in Figure 3, this interference creates potential congestion (shaded area) where the two activities have both spatial and temporal overlaps.

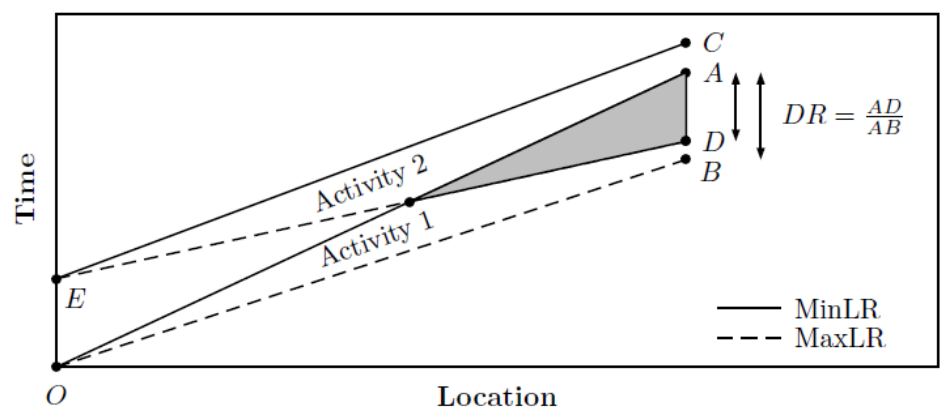

Figure 3: Space-time overlapping between activities

The equation below (Eq. 4) measures this overlapping impact as a dependency ratio between consecutive activities (refer to DR in Figure 3). The ratio is clearly controlled by both the progress and the learning rates.

$$
\begin{aligned}
D R= & \frac{A D}{A B} \\
= & \frac{Y_{P \min }(P)-Y_{P \max }(X)}{Y_{P \min }(P)-Y_{P \max }(P)} \\
= & \frac{1}{P_{\min (P)}}-\frac{1}{P_{\min (X)}} \times k_{1}^{\frac{1 \log L R x}{\log 2}} \\
P_{\min (P)} & \frac{1}{P_{\min (P)}} \times k_{2}^{\frac{\log L R P}{\log 2}} \\
= & \frac{P_{\min (X)}-P_{\min (P)} \times k_{1}^{\frac{\log L R x}{\log 2}}}{P_{\min (X)}-P_{\min (X)} \times k_{2}^{\frac{\log L R P}{\log 2}}}
\end{aligned}
$$

Where: $\mathrm{k}_{1}$ and $\mathrm{k}_{2}$ are the algebraic midpoints of the total number of iterations or repetitions in the project; $\mathrm{X}$ is the current activity and $\mathrm{P}$ is its corresponding predecessor; LRx and LRp are the maximum learning rates of the current activity and its predecessor respectively; $\mathrm{Y}_{\mathrm{pmin}}(\mathrm{P})$ is the ordinate of point $\mathrm{A} ; \mathrm{Y}_{\mathrm{pmax}}(\mathrm{X})$ is the ordinate of point $\mathrm{D}$; and $\mathrm{Y}_{\mathrm{pmax}}(\mathrm{P})$ is the ordinate of point $\mathrm{B}$.

Therefore, an activity with a low dependency ratio is less subject to creating congestion with its predecessors and accordingly requires a smaller percentage reduction in its space-time float. Conversely, a greater percentage reduction is witnessed for activities with higher dependency ratios as they are more likely to interfere with their predecessors. Moreover, if the current activity has more than one predecessor, only the maximum dependency ratio is considered as the input and if the maximum productivity rate of the current activity doesn't interfere with the space-time float of its predecessors, the dependency ratio becomes zero.

\section{Conclusion}

It has been always stated that learning curves can significantly impact construction labor productivity. However, this impact can become negative in linear projects or horizontal repetitive projects where the deviations from planned productivity rates may lead to potential congestion. Some activities may undergo a maximum productivity improvement due to learning, while others might witness no improvement. In most cases, this phenomenon can lead to space-time overlapping between activities, thereby causing project delays. Moreover, the flexibility of the activity itself in addition to the activity influence on the project network can also impact the inherent schedule uncertainties. Therefore, given the three aforementioned uncertainty dimensions, the range of productivities, defined by minimum learning (zero-learning) and maximum learning rates, needs to be reduced to an allowable learning development range for each activity. As such, this paper presented a fuzzy-based system targeted at quantifying the learning development range for each activity of repetitive construction projects. The proposed fuzzy-based system takes in the uncertainty 
Mohammad Rahal, Hiam Khoury/ Proceedings of the Creative Construction Conference (2019) 074 https://doi.org/10.3311/CCC2019-074

dimensions as inputs and generates the minimum percentage reduction in the activities' productivity range as output. These percentages will, in future work, be input into a time-cost optimization framework that aims at minimizing congestion in linear schedules while considering the learning curve effect.

\section{References}

Ammar, M. A. (2012). LOB and CPM integrated method for scheduling repetitive projects. Journal of Construction Engineering and Management, 139(1), 44-50. https://doi.org/10.1061/(ASCE)CO.1943-7862.0000569

Ammar, M. A., \& Abdel-Maged, A. F. (2018). Modeling of LOB scheduling with learning development effect. International Journal of Construction Management, 18(6), 517-526. https://doi.org/10.1080/15623599.2017.1357350

Ammar, M. A., \& Elbeltagi, E. (2001). Algorithm for determining controlling path considering resource continuity. Journal of Computing in Civil Engineering, 15(4), 292-298. https://doi.org/10.1061/(ASCE)0887-3801(2001)15:4(292)

Arditi, D., Tokdemir, O. B., \& Suh, K. (2001). Effect of learning on line-of-balance scheduling. International journal of project management, 19(5), 265-277. https://doi.org/10.1016/S0263-7863(99)00079-4

Arditi, D., Tokdemir, O. B., \& Suh, K. (2002). Challenges in line-of-balance scheduling. Journal of Construction Engineering and Management, 128(6), 545-556. https://doi.org/10.1061/(ASCE)0733-9364(2002)128:6(545)

Chan, A. P., Chan, D. W., \& Yeung, J. F. (2009). Overview of the application of "fuzzy techniques" in construction management research. Journal of Construction Engineering and Management, 135(11), 1241-1252. http://dx.doi.org/10.1061/(ASCE)CO.1943-7862.0000099

Elbeltagi, E., Elkassas, E., Rasheed, I. A., \& Al-Tawil, S. (2007). Scheduling and Cost Optimization of Repetitive Projects Using Genetic Algorithms. ijm, 500, 1. https://doi.org/10.1201/b13165-69

Garibaldi, J. M., \& John, R. I. (2003). Choosing membership functions of linguistic terms. Paper presented at the The 12th IEEE International Conference on Fuzzy Systems, 2003. FUZZ'03. https://doi.org/10.1109/FUZZ.2003.1209428

Hajdu, M. (2015). History and some latest developments of precedence diagramming method. International Journal of Organization, Technology and Management in Construction, 7(2), 1302-1314.

Hajdu, M. (2016). PDM time analysis with continuous and point-to-point relations: Calculations using an artificial example. Procedia Engineering, 164, 57-67. https://doi.org/10.1016/j.proeng.2016.11.592

Hamade, R. F., Jaber, M. Y., \& Sikström, S. (2009). Analyzing CAD competence with univariate and multivariate learning curve models. Computers \& Industrial Engineering, 56(4), 1510-1518. https://doi.org/10.1016/j.cie.2008.09.025

Hinze, J., \& Olbina, S. (2009). Empirical analysis of the learning curve principle in prestressed concrete piles. Journal of Construction Engineering and Management, 135(5), 425-431. https://doi.org/10.1061/(ASCE)CO.1943-7862.0000004

Jaber, M. Y. (2016). Learning curves: Theory, models, and applications: CRC Press.

Jarkas, A., \& Horner, M. (2011). Revisiting the applicability of learning curve theory to formwork labour productivity. Construction Management and Economics, 29(5), 483-493. https://doi.org/10.1080/01446193.2011.562911

Jarkas, A. M. (2010). Critical investigation into the applicability of the learning curve theory to rebar fixing labor productivity. Journal of Construction Engineering and Management, 136(12), 1279-1288. http://dx.doi.org/10.1061/(ASCE)CO.1943-7862.0000236

Lam, K., Lee, D., \& Hu, T. (2001). Understanding the effect of the learning-forgetting phenomenon to duration of projects construction. International journal of project management, 19(7), 411-420. https://doi.org/10.1016/S0263-7863(00)00025-9

Mályusz, L., \& Pém, A. (2013). Prediction of the learning curve in roof insulation. Automation in Construction, 36, 191-195. https://doi.org/10.1016/j.autcon.2013.04.004

Moselhi, O., \& El-Rayes, K. (1993). Scheduling of repetitive projects with cost optimization. Journal of Construction Engineering and Management, 119(4), 681-697. https://doi.org/10.1061/(ASCE)0733-9364(1993)119:4(681)

Panas, A., \& Pantouvakis, J. P. (2013). Simulation-based and statistical analysis of the learning effect in floating caisson construction operations. Journal of Construction Engineering and Management, 140(1), 04013033. http://dx.doi.org/10.1061/(ASCE)CO.1943-7862.0000782

Sivanandam, S., Sumathi, S., \& Deepa, S. (2007). Introduction to fuzzy logic using MATLAB (Vol. 1): Springer.

Suhail, S. A., \& Neale, R. H. (1994). CPM/LOB: New methodology to integrate CPM and line of balance. Journal of Construction Engineering and Management, 120(3), 667-684. https://doi.org/10.1061/(ASCE)0733-9364(1994)120:3(667)

Thabet, W. Y., \& Beliveau, Y. J. (1994). HVLS: Horizontal and vertical logic scheduling for multistory projects. Journal of Construction Engineering and Management, 120(4), 875-892. https://doi.org/10.1061/(ASCE)0733-9364(1994)120:4(875)

Tokdemir, O. B., Arditi, D., \& Balcik, C. (2006). ALISS: advanced linear scheduling system. Construction Management and Economics, 24(12), 1253-1267. https://doi.org/10.1080/01446190600953706

Wang, C. (2015). A study of membership functions on mamdani-type fuzzy inference system for industrial decision-making.

Wong, P. S., On Cheung, S., \& Hardcastle, C. (2007). Embodying learning effect in performance prediction. Journal of Construction Engineering and Management, 133(6), 474-482. https://doi.org/10.1061/(ASCE)0733-9364(2007)133:6(474)

Wright, T. P. (1936). Learning curve. Journal of the Aeronautical Sciences, 3(1), 122-128. 
Mohammad Rahal, Hiam Khoury/ Proceedings of the Creative Construction Conference (2019) 074

https://doi.org/10.3311/CCC2019-074

Yang, L., Fu, Y., Li, S., Huang, B., \& Peng, T. (2008). A buffer sizing approach in critical chain scheduling with attributes dependent. Paper presented at the 2008 4th International Conference on Wireless Communications, Networking and Mobile Computing. https://doi.org/10.1109/WiCom.2008.1805

Zahran, K., Nour, M., \& Hosny, O. (2016). The effect of learning on line of balance scheduling: Obstacles and potentials. International Journal of Engineering Science, 6(4), 3831-3841. http://dx.doi.org/10.1016/S0263-7863(99)00079-4

Zhang, L., Zou, X., \& Kan, Z. (2014). Improved strategy for resource allocation in repetitive projects considering the learning effect. Journal of Construction Engineering and Management, 140(11), 04014053. https://doi.org/10.1061/(ASCE)CO.1943-7862.0000896 